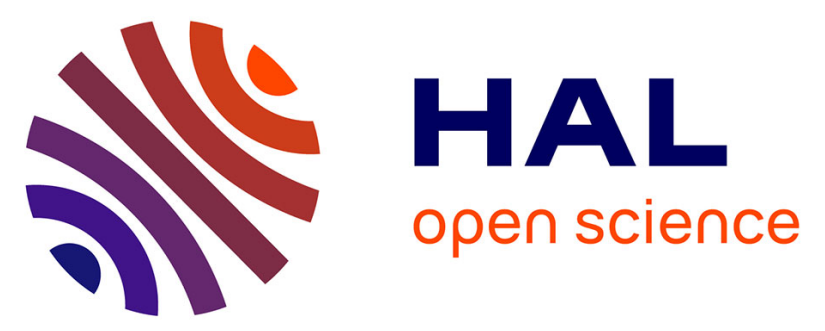

\title{
Prevalence of doravirine-associated resistance mutations in HIV-1-infected antiretroviral-experienced patients from two large databases in France and Italy
}

Cathia Soulie, Maria Mercedes Santoro, Alexandre Storto, Basma Abdi, Charlotte Charpentier, Daniele Armenia, Aude Jary, Federica Forbici, Ada Bertoli, William Gennari, et al.

\section{To cite this version:}

Cathia Soulie, Maria Mercedes Santoro, Alexandre Storto, Basma Abdi, Charlotte Charpentier, et al.. Prevalence of doravirine-associated resistance mutations in HIV-1-infected antiretroviral-experienced patients from two large databases in France and Italy. Journal of Antimicrobial Chemotherapy, 2020, 75 (4), pp.1026-1030. 10.1093/jac/dkz553 . hal-02988121

\section{HAL Id: hal-02988121 \\ https://hal.sorbonne-universite.fr/hal-02988121}

Submitted on 4 Nov 2020

HAL is a multi-disciplinary open access archive for the deposit and dissemination of scientific research documents, whether they are published or not. The documents may come from teaching and research institutions in France or abroad, or from public or private research centers.
L'archive ouverte pluridisciplinaire HAL, est destinée au dépôt et à la diffusion de documents scientifiques de niveau recherche, publiés ou non, émanant des établissements d'enseignement et de recherche français ou étrangers, des laboratoires publics ou privés. 
Running title: Doravirine resistance in HIV-1 treated patients

5

6 Cathia SOULIE ${ }^{1 *}$, Maria Mercedes SANTORO ${ }^{2}$, Alexandre STORTO ${ }^{3}$, Basma ABDI ${ }^{1}$, Charlotte

CHARPENTIER ${ }^{3,4}$, Daniele ARMENIA ${ }^{2,5}$, Aude Jary ${ }^{1}$, Federica FORBICl ${ }^{6}$, Ada BERTOLI ${ }^{2}$, William

8 GENNARI $^{7}$, Massimo ANDREONI ${ }^{8}$, Cristina MUSSINI ${ }^{7}$, Andrea ANTINORI ${ }^{6}$, Carlo Federico

PERNO $^{9}$, Vincent CALVEZ ${ }^{1}$, Francesca CECCHERINI-SILBERSTEIN ${ }^{2}$, Diane DESCAMPS 3,4 , Anne-

Genevieve MARCELIN ${ }^{1}$

${ }^{1}$ Sorbonne Université, INSERM, UMR_S 1136 Pierre Louis Institute of Epidemiology and Public Health, AP-HP, Hôpital Pitié Salpêtrière, laboratoire de virologie, Paris, France; ${ }^{2}$ University of

Rome "Tor Vergata", Department of Experimental Medicine, Rome, Italy; ${ }^{3}$ Université de Paris, INSERM UMR 1137 IAME, F-75018 Paris, France; ${ }^{4}$ Laboratoire de Virologie, AP-HP, Hôpital

Bichat-Claude Bernard, F-75018 Paris, France; ${ }^{5}$ UniCamillus, Saint Camillus International University of Health Sciences, Rome, Italy; ${ }^{6}$ National Institute for Infectious Diseases L.

Spallanzani, IRCCS, Rome, Italy; ${ }^{7}$ Modena University Hospital, Clinic of Infectious Diseases,

Modena, Italy; ${ }^{8}$ University Hospital of Rome Tor Vergata, Department of Clinical Infectious

Diseases, Rome, Italy; ${ }^{9}$ University of Milan, Department of Oncology and Hemato-oncology, Milan, Italy. 
23 Corresponding author: Dr Cathia Soulié, Laboratoire de Virologie-CERVI, Hôpital Pitié

24 Salpêtrière 45-83 Bd de l'hôpital 75013 Paris, France. Phone: 331421758 42. Fax: 33142

251774 11. Email: cathia.soulie-ext@aphp.fr

26 Word count: 1912.

27 
ABSTRACT

30

31 Objectives: Doravirine, a novel NNRTI, selects for specific mutations in vitro, including

32 mutations at reverse transcriptase (RT) positions 106, 108, 188, 227, 230 and 234. The aim of this study was to examine the prevalence of doravirine-associated resistance mutations in HIV-1-infected antiretroviral-experienced patients.

Methods: Doravirine-associated resistance mutations identified in vitro or in vivo were studied in a set of 9199 HIV-1 RT sequences from HIV-1 antiretroviral-experienced patients, including 381 NNRTI-failing patients in France and Italy between 2012 and 2017. The following mutations were considered as resistance mutations: V106A/M, V108I, Y188L, G190S, F227C/L/V, M230I/L, L234I, P236L, K103N+Y181C, K103N+P225H, K103N+L100I.

Results: The frequencies of doravirine-associated resistance mutations (total set versus NNRTI-failing patients) were V106A/M (0.8\% versus $2.6 \%)$, V108I (3.3\% versus $9.2 \%), \mathrm{Y} 188 \mathrm{~L}$ (1.2\% versus $2.6 \%)$, G190S (0.3\% versus $2.1 \%), \mathrm{F} 227 \mathrm{C} / \mathrm{L} / \mathrm{V}$ (0.5\% versus $1.8 \%), \mathrm{M} 230 \mathrm{I} / \mathrm{L}(2.8 \%$ versus $0 \%), \mathrm{L} 234 \mathrm{I}(0.1 \%$ versus $0.5 \%), \mathrm{K} 103 \mathrm{~N}+\mathrm{Y} 181 \mathrm{C}(3.9 \%$ versus $3.9 \%), \mathrm{K} 103 \mathrm{~N}+\mathrm{P} 225 \mathrm{H}(2.9 \%$ versus $4.7 \%)$ and $\mathrm{K} 103 \mathrm{~N}+100 \mathrm{I}(1.7 \%$ versus $3.9 \%)$ with a significant higher proportion of these mutations in the NNRTI-failing group ( $p<0.05$ ), except for $\mathrm{M} 230 \mathrm{I} / \mathrm{L}$ and $\mathrm{K} 103 \mathrm{~N}+\mathrm{Y} 181 \mathrm{C}$. The overall prevalence of sequences with at least 1 doravirine-associated resistance mutation was $12.2 \%$ and $34.9 \%$ in total set and NNRTI-failing patients $(p<0.001)$, respectively. In comparison, the prevalence of common NNRTI mutations V90I, K101E/P, K103N/S, E138A/G/K/Q/R/S, Y181C/I/V, G190A/E/S/Q were higher (8.9\%, 7.9\%, 28.6\%, 12.6\%, 14.2\%, 8.9\%, respectively). 
50 Conclusions: These results suggest that doravirine resistance in antiretroviral-experienced

51 patients generally and specifically among NNRTI-failing patients is lower than resistance to

52 other NNRTIs currently used, confirming its distinguishing resistance pattern.

53

54

55 


\section{INTRODUCTION}

57 Non-nucleoside reverse transcriptase inhibitors (NNRTIs) are a major component of the antiretroviral treatment for HIV patients, as they were the third recommended agent in the WHO and European guidelines, and until recently in US guidelines. ${ }^{1-3}$ First generation NNRTIs, efavirenz and nevirapine, have a low-level genetic barrier to resistance and consequently the prevalence of HIV-1 resistance to NNRTIs is the highest one of the several classes of antiretrovirals, in HIV naïve as well in treated patients. ${ }^{4-6}$ Then, new NNRTIs drugs retaining antiretroviral activity against viruses with K103N, E138K, Y181C and G190A, the most prevalent NNRTI mutations, are needed.

Two large phase 3 studies have demonstrated the efficacy of doravirine, a new NNRTI, in a population of naïve HIV patients in comparison to efavirenz (DRIVE-AHEAD) or boosted darunavir (800/100) (DRIVE-FORWARD) in combination with two nucleoside reverse transcriptase inhibitors (NRTIS). ${ }^{7-9}$ In the DRIVE-SHIFT trial, the switch to doravirine/lamivudine/tenofovir disoproxil fumarate maintained virological suppression through 48 weeks. ${ }^{10}$

The doravirine resistance profile is distinct from other NNRTIs with the in vitro selection of mutations at reverse transcriptase (RT) positions 106, 108, 188, 227, 230, 234 and 236. ${ }^{11-14}$ In vivo, the evidenced resistance mutation profiles were concordant: Y188L; V106I+F227C; V106I/V+H221Y+F227C; F227C; V106A+P225H+Y318Y/F; V106T/M, F227C/R; Y318F/Y. ${ }^{7-9}$ We aimed to study the prevalence of doravirine-associated resistance mutations in HIV-1infected antiretroviral-experienced patients, and especially in NNRTI-failing patients to investigate whether the previous NNRTI use could impair doravirine activity. 
Resistance genotypic tests were performed at five reference laboratories, 2 in Paris (PitiéSalpêtrière and Bichat Claude Bernard hospitals) and 3 in Italy (University/Polyclinic of Rome "Tor Vergata", INMI Spallanzani-IRCCS, and Modena Hospital). A total of 9199 HIV-1 RT sequences obtained between 2012 and 2017 from HIV-1 antiretroviral-experienced patients in routine clinical care were analysed. A follow-up of HIV viral load about 3 to 6 months; in case of 2 consecutive viral loads $>50$ copies $/ \mathrm{mL}$, a resistance genotypic testing was performed on the second viral load. Among this set of sequences, 381 sequences were originated from NNRTI-failing patients (efavirenz, $n=189$; etravirine, $n=32$; nevirapine, $n=66$; rilpivirine, $n=94$ ). The following mutations identified in vitro or in vivo were considered as doravirine-associated mutations: RT V106A/M, V108I, Y188L, F227C/L/V, M230I/L, L234I, P236L, K103N+P225H, K103N+L100I. ${ }^{8,11-14} \mathrm{~K} 103 \mathrm{~N}+\mathrm{Y} 181 \mathrm{C}$ and G190S were also considered in our analysis, as they are known to confer resistance to other NNRTIs. NNRTIs mutations associated with resistance to efavirenz, rilpivirine, nevirapine and etravirine were those listed in the ANRS algorithm (table of rules 2018; www.hivfrenchresistance.org), in the IAS list 2018 (www.iasusa.org) and in the Stanford HIV drug resistance database (https://hivdb.stanford.edu/drsummary/resistance-notes/NNRTI/). Namely, efavirenz: L100I, K101E/P, K103N/S, V106A/M, V108I, Y181C/I/V, Y188C/H/L, G190A/E/S, P225H, M230L; etravirine: V90I, A98G, L100I, K101E/H/P, V106I, E138A/G/K/Q, V179D/F/T, Y181C/I/V, G190A/E/S, M230L; nevirapine: L100I, K101E/P, K103N/S, V106A/M, V108I, Y181C/I/V, Y188C/H/L, G190A/E/S, M230L; rilpivirine: L100I, K101E/P, E138A/G/K/Q/R, V179L, Y181C/I/V, Y188L, G190A/E/S, H221Y, F227C, M230I/L.

Resistance interpretation was made using the Smartgene ${ }^{\circledR}$ Integrated Database Network System (SmartGene, Switzerland; http://www.smartgene.com) according to the Stanford (https://hivdb.stanford.edu/) and the ANRS (table of rules 2018; 
www.hivfrenchresistance.org) algorithms. Resistance and possible resistance were grouped as resistance.

Subtype was determined on the basis of the RT and protease coding regions by Smartgene algorithm (Smartgene ${ }^{\circledR}$, Switzerland) or by phylogenetic analyses, using reference sequences of HIV-1 subtypes and circulating recombinant forms (CRF) from the Los Alamos Database (https://www.hiv.lanl.gov/content/sequence/HIV/mainpage.html). Between-group comparisons were carried out using Fisher's exact test.

\section{RESULTS}

\section{Distribution of HIV-1 subtypes}

Among the 9199 sequences, the distribution of subtypes was: $45.3 \%$ B, 27.3\% CRF02_AG, 3.7 \% A1, 2.5\% C, 1.7\% CRF06_cpx and 19.5\% other various non-B. Among the 381 sequences of NNRTI-failing patients, 252 (66.1\%) were infected with a B subtype and 129 (33.9\%) with a non-B subtype. The distribution of the subtype (B versus non-B) was statistically different between the all and NNRTI-failing group $(p<0.001)$.

\section{Prevalence of doravirine and other NNRTIs resistance associated mutations}

Analyzing the overall dataset of HIV-1 antiretroviral-experienced patients, the most frequent doravirine resistance associated mutations were V106A/M 0.8\% ( $n=77)$, V108I 3.3\% ( $n=307)$, Y188L 1.2\% ( $n=107)$, G190S 0.3\% ( $n=24)$, F227C/L/V 0.5\% ( $n=49), M 230 I / L 2.8 \%$ ( $n=256), L 234 I$ $0.1 \%(n=13), P 236 L$ 0\% ( $n=0), K 103 N+Y 181 C 3.9 \%(n=361), K 103 N+P 225 H 2.9 \%(n=264)$ and $\mathrm{K} 103 \mathrm{~N}+100 \mathrm{I} 1.7 \%(\mathrm{n}=156)$ (figure 1). The prevalence of $\mathrm{M} 230 \mathrm{I} / \mathrm{L}$ and $\mathrm{K} 103 \mathrm{~N}+\mathrm{L} 100 \mathrm{I}$ was higher 
128 for the HIV-1 B subtype than non-B subtypes (3.3\% versus $2.4 \%, p=0.009$ and $2.6 \%$ versus

$1291.0 \%, p<0.001$, respectively), in contrast to $\mathrm{K} 103 \mathrm{~N}+\mathrm{P} 225 \mathrm{H}(1.8 \%$ versus $3.7 \%, \mathrm{p}<0.001)$.

130 In comparison, the prevalence of common NNRTIs mutations V90I, A98G, L100I, K101E/P, $131 \mathrm{~K} 103 \mathrm{~N} / \mathrm{S}, \mathrm{E} 138 \mathrm{~A} / \mathrm{G} / \mathrm{K} / \mathrm{O} / \mathrm{R}, \mathrm{V} 179 \mathrm{D} / \mathrm{F} / \mathrm{T}, \mathrm{Y} 181 \mathrm{C} / \mathrm{I} / \mathrm{V}, \mathrm{Y} 188 \mathrm{C} / \mathrm{H} / \mathrm{L}, \mathrm{G} 190 \mathrm{~A} / \mathrm{E} / \mathrm{S}, \mathrm{T} 225 \mathrm{H}$ were 6.3\% 132 (580), 2.5\% (231), 1.0\% (94), 2.4\% (219), 10.2\% (934), 10.9\% (1001), 1.5\% (137), 5.7\% (521), $1331.7 \%(153), 2.8 \%(258), 1.4 \%(130)$, respectively (figure 1). Some mutations were more 134 frequent in HIV-1 B subtype [L100I (1.6\% versus $0.6 \%, p<0.001), E 138 A / G / K / Q / R(14.3 \%$ versus $1358.0 \%, \mathrm{p}<0.001), \mathrm{V} 179 \mathrm{D} / \mathrm{F} / \mathrm{T}(2.0 \%$ versus $1.1 \%, \mathrm{p}<0.001), \mathrm{G} 190 \mathrm{~A} / \mathrm{E} / \mathrm{S}$ (2.9\% versus $2.7 \%$, $136 p=0.004)$ ] or in HIV-1 non-B subtypes [V90I (4.2\% versus $8.0 \%, p<0.001)$, A98G $(1.8 \%$ versus $1373.1 \%, \mathrm{p}<0.001), \mathrm{K} 103 \mathrm{~N} / \mathrm{S}(8.7 \%$ versus $11.3 \%, \mathrm{p}<0.001)$, and $\mathrm{T} 225 \mathrm{H}(1.2 \%$ versus $1.6 \%$, $138 p<0.001)]$. There was no difference between $B$ and non-B subtypes for E138K (4.0\% versus $1393.1 \%, p=0.407)$.

140

\section{Resistance to doravirine and other NNRTIs}

142 The overall prevalence of sequences with at least 1 doravirine resistance associated mutation 143 was $12.2 \%(n=1119)$. Considering the ANRS algorithm, 5.6\% $(n=512)$ of sequences were 144 considered resistant to doravirine. In comparison, the prevalence of sequences considered as 145 resistant were significantly higher for efavirenz $(18.8 \%, n=1725)$, etravirine $(8.4 \%, n=776)$, 146 nevirapine (17.9\%, $n=1647)$ and rilpivirine (22.3\%, $n=2050),(p<0.001)$ (figure $2 A)$. Similarly, 147 with the Stanford algorithm, the prevalence of sequences considered as resistant to doravirine 148 was $16.0 \%(n=1468)$, and lower than those for efavirenz $24.8 \%(n=2277)$, etravirine $24.6 \%$ 149 ( $n=2267)$, nevirapine 24.9\% ( $n=2294)$ and rilpivirine $24.7 \%(n=2269)(p<0.001)$ (figure 2B). 
153 Analyzing the NNRTI-failing patients, among the doravirine resistance associated mutations, 154 the most frequent mutations were V106A/M 2.6\% (10), V108I 9.2\% (35), Y188L 2.6\% (10),

155

156

157

158 prevalence of sequences considered as resistant to other NNRTIs by ANRS algorithm: $36.5 \%$

173 ( $n=139)$ were genotypically resistant to nevirapine $(p<0.001), 51.7 \%(n=197)$ to efavirenz 174 G190S 2.1\% (8), F227C/L/V 1.8\% (7), M230I/L 0\% (0), L234I 0.5\% (2), P236L 0\%, K103N+Y181C 3.9\% (15), $\mathrm{K} 103 \mathrm{~N}+\mathrm{P} 225 \mathrm{H} 4.7 \%$ (18) and $\mathrm{K} 103 \mathrm{~N}+\mathrm{L} 100 \mathrm{I} 3.9 \%$ (15) (figure 1). The following mutations are statistically more prevalent $(p<0.05)$ in the NNRTI-failing group comparing to the whole set of sequences: V106A/M, V108I, Y188L, G190S, F227C/L/V and K103N+L100I. Only $\mathrm{M} 230 \mathrm{I} / \mathrm{L}$ was statistically more prevalent in the whole group than in the NNRTI-failing group ( $p<0.001$ ) (figure 1). Furthermore, the mutation F227C/L/V was less frequent in B versus non-B subtypes $(0.8 \%$ versus $3.9 \%, p=0.047)$.

In comparison, the prevalence of common NNRTIs mutations V90I, A98G, L100I, K101E/P, K103N/S, E138A/G/K/Q/R, V179D/F/T, Y181C/I/V, Y188C/H/L, G190A/E/Q/S, T225H were 8.9\% ( $n=34), 3.4 \%(n=13), 4.2 \%(n=16), 7.9 \%(n=30), 28.6 \%(n=109), 12.6 \%(n=48), 5.2 \%$ $(n=20), 14.2 \%(n=54), 4.7 \%,(n=18), 8.9 \%(n=34), 5.2 \%(n=20)$, respectively (figure 1$)$. No association was observed between these common mutations and HIV subtype.

\section{Resistance in the NNRTI-failing group}

The overall prevalence of sequences with at least 1 doravirine resistance associated mutations was $34.9 \%(n=133)$. Considering the ANRS algorithm, $18.1 \%(n=69)$ of sequences were considered as resistant to doravirine. This prevalence was significantly lower than the $(p<0.001), 21.9 \%(n=88)$ to etravirine $(p=0.107)$ and $55.6 \%(n=212)$ to rilpivirine $(p<0.001)$ 
175 (Figure 1A). With the Stanford algorithm, the resistance to doravirine was $42.0 \%(n=160)$ and

176 not different from etravirine and rilpivirine resistance, whereas the resistance to the first 177 generation NNRTIs was higher: efavirenz $(52.0 \%, n=209, p<0.001)$ and nevirapine (56.2\%, $178 n=214, p<0.001$ ) (Figure 1B).

179

\section{DISCUSSION}

Our study evidenced a low prevalence of doravirine resistance associated mutations in HIV-1infected antiretroviral-treated patients in Italy and France. This prevalence was significantly

184 lower than those for other NNRTIs in use, especially first generation NNRTIs.

In this study, the proportion of non-B subtypes was high (54.6\%), with a large variety of

186 subtypes, and slightly higher than in our previous study on doravirine resistance in HIV-1

187 antiretroviral-naïve patients (47.0\%). ${ }^{15}$ However, it was similar to the prevalence of non-B 188 subtypes in antiretroviral-naïve chronically HIV-infected patients in 2015/2016 in France 189 (54.8\%). 5

190 As expected, the prevalence of the resistance associated to doravirine and other NNRTIs was 191 higher in the population of HIV-1 antiretroviral treated patients than in our previous study 192 that showed the rare occurrence of doravirine resistance associated mutations in HIV-1 193 infected antiretroviral naïve patients ( $n=137 / 9764,1.4 \%) .{ }^{15}$ For K103N, Y181C and E138A/K 194 mutations, their prevalences observed in this study were more consequential than in the most 195 recent French nationwide study in treated patients with a confirmed viral load $>50$ copies $/ \mathrm{mL}$. 1965

197 In the DRIVE clinical trials conducted in HIV-1 antiretroviral naïve patients, the evidenced 198 resistance mutation profiles at failure were as follow: Y188L; V016I+F227C; 
Globally and except for the single Y318F not studied here, all these doravirine mutations were

201 present at a low percentage, even in the NNRTI-failing patients in our study.

In DRIVE-SHIFT, conducted in virologically suppressed patients, no doravirine resistance associated mutations were evidenced in patients achieving the protocol-defined virological failure. Of note, 24 participants had a virus with baseline NNRTI mutations (K103N, Y181C and G190A) and 23/24 who switched to doravirine/lamivudine/tenofovir disoproxil fumarate remained suppressed during the 48 week follow-up. ${ }^{16}$ This suggests, that the most frequent NNRTI mutations at RT mutation positions 103, 181 and 190, should probably not impact the doravirine activity in vivo. $\mathrm{K} 103 \mathrm{~N}+\mathrm{Y} 181 \mathrm{C}$ and G190S, although not DOR-associated resistant substitutions, were included in our analysis as they confer resistance to other NNRTIs. In our 210 study, the prevalence of the K103N+Y181C and G190S was low and did not impact the global 211 resistance of doravirine.

212 Some small significant differences were observed in the present study for the prevalence of 213 some doravirine mutations, according to the HIV-1 subtypes (M230I/L, K103N+L100I more 214 frequent in B subtype and $\mathrm{K} 103 \mathrm{~N}+\mathrm{P} 225 \mathrm{H}$ more frequent in non-B subtypes). In another study, 215 it has been shown that Y188L and V106M were more frequent in C subtype while V106A was 216 less frequent in non-B subtypes. ${ }^{17}$

217 One limitation of the study is the relatively low number of NNRTI value. However, when the 218 resistance test was performed in case of virological failure for these antiretroviral treated 219 patients, more patients had probably previously been exposed to NNRTIs.

220 The results of interpreting doravirine resistance were different according to ANRS or Stanford 221 algorithms (18.1\% versus 42\%) in the NNRTI-failing group. It could be explained together by 222 differences in the set mutations list for a same RT position and also in the number of 
223 considered positions. For example, the following RT mutations are not taking into account in

224 the ANRS algorithm: L100I, K101E/P, V106I, Y181C/I/V, P225H, F227C, L234I.

225 Our study shows, according to the Stanford algorithm, 42\% of doravirine resistance in the 226 NNRTI-failing group which was higher than recently evidenced in NNRTI-experienced patients 227 in another study (18.8\%). ${ }^{17}$ Several factors could explain this difference. The studied 228 doravirine mutations were not strictly similar between the two studies. Indeed, we 229 investigated a larger set of mutations with the inclusion of mutations G190S, F227C/V, M230I, $230 \mathrm{~L} 234 \mathrm{I}, \mathrm{P} 236 \mathrm{~L}, \mathrm{~K} 103 \mathrm{~N}+\mathrm{Y} 181 \mathrm{C}$ and $\mathrm{K} 103 \mathrm{~N}+\mathrm{L} 100 \mathrm{I}$, as well as the $\mathrm{F} 227 \mathrm{C}$ and the $\mathrm{L} 234 \mathrm{I}$ alone and 231 not only in association with the V106A/M.

232 In conclusion, these results suggest that doravirine resistance in antiretroviral-experienced 233 patients generally and specifically among NNRTI-failing patients is significantly lower than 234 resistance to other NNRTIs currently used, confirming its distinguishing resistance pattern. In 235 addition, these results are reassuring in the perspective of the use of doravirine in 236 antiretroviral-treated patients and exposed to previous use of NNRTIs after availability of a 237 genotype. 


\section{ACKNOWLEDGMENTS}

240 A part of this work was presented at the $17^{\text {th }}$ EU Meeting on HIV \& Hepatitis, Rome, Italy, 2019 241 (abstract 9).

242 We thank all of the patients and all of our clinical and laboratory colleagues for providing data

243 for this study across the two countries.

244

\section{FUNDING}

246 This work was supported by 'Agence Nationale de recherche sur le SIDA et les hépatites

247 virales' (ANRS), MSD, the Italian Ministry of Education, University and Research (MIUR)

248 (Bandiera InterOmics Protocollo PB05 1_) and an unrestricted grant from The Italian

249 Foundation for Antiviral Studies and Research (AVIRALIA FOUNDATION).

250

251 TRANSPARENCY DECLARATIONS

252 A. G. M. and C. C. have received honoraria and travel grants from ViiV Healthcare, Janssen-

253 Cilag, Gilead Sciences and MSD. M. M. S. has received funds for attending symposia, speaking

254 and organizing educational activities from ViiV and Janssen-Cilag.

255 All other authors: none to declare. 


\section{BIBLIOGRAPHY}

258

259 1. WHO. 2016 consolidated guidelines on the use of antiretroviral drugs for treating and 260 preventing HIV infection. 2016. Available at: http://www.who.int/hiv/pub/arv/arv-2016/en/.

261 2. EACS European, AIDS Clinical Society. European Guidelines for treatment of HIV-positive 262 adults in Europe. 2017. Available at: https://www.eacsociety.org/files/2019 guidelines$263 \quad 10.0$ final.pdf

264 3. Department of Health and Human services. Panel on antiretroviral guidelines for adults and 265 adolescents. 2018. Available at:

\section{https://aidsinfo.nih.gov/contentfiles/lvguidelines/adultandadolescentgl.pdf}

267 4. Assoumou L, Charpentier C, Recordon-Pinson P, et al. Prevalence of HIV-1 drug resistance 268 in treated patients with viral load $>50$ copies $/ \mathrm{mL}$ : a 2014 French nationwide study. J 269 Antimicrob Chemother 2017; 72: 1769-73.

270 5. Assoumou L, Bocket L, Pallier C, et al. Stable prevalence of transmitted drug resistance

271 mutations and increased circulation of non-B subtypes in antiretroviral-naive chronically HIV-

272 infected patients in 2015/2016 in France. J Antimicrob Chemother 2019; 74 (5): 1417-1424.

273 6. Brenner BG, Ibanescu R-I, Hardy I, et al. Genotypic and Phylogenetic Insights on Prevention 274 of the Spread of HIV-1 and Drug Resistance in 'Real-World' Settings. Viruses 2017; 10: (1) pii: 275 E10. 
276 7. Molina J-M, Squires K, Sax PE, et al. Doravirine versus ritonavir-boosted darunavir in 277 antiretroviral-naive adults with HIV-1 (DRIVE-FORWARD): 48-week results of a randomised, 278 double-blind, phase 3, non-inferiority trial. Lancet HIV 2018; 5 (5): e211-220.

8. Orkin C, Squires KE, Molina J-M, et al. Doravirine/Lamivudine/Tenofovir Disoproxil 280 Fumarate is Non-inferior to Efavirenz/Emtricitabine/Tenofovir Disoproxil Fumarate in 281 Treatment-naive Adults With Human Immunodeficiency Virus-1 Infection: Week 48 Results of 282 the DRIVE-AHEAD Trial. Clin Infect Dis 2019; 68: 535-44.

283 9. Colombier M-A, Molina J-M. Doravirine: a review. Curr Opin HIV AIDS 2018; 13: 308-14.

284 10. Kumar PN, Johnson M, Molina JM, et al. Switch to Doravirine/Lamivudine/Tenofovir 285 Disoproxil Fumarate (DOR/3TC/TDF) Maintains Virologic Suppression Through 48 Weeks: 286 Results of the DRIVE-SHIFT Trial. In: Vol LB2. San Francisco, CA, 2018.

287 11. Lai M-T, Feng M, Falgueyret J-P, et al. In vitro characterization of MK-1439, a novel HIV-1 288 nonnucleoside reverse transcriptase inhibitor. Antimicrob Agents Chemother 2014; 58: 165228963.

290 12. Feng M, Sachs NA, Xu M, et al. Doravirine Suppresses Common Nonnucleoside Reverse 291 Transcriptase Inhibitor-Associated Mutants at Clinically Relevant Concentrations. Antimicrob 292 Agents Chemother 2016; 60: 2241-7.

293 13. Feng M, Wang D, Grobler JA, et al. In vitro resistance selection with doravirine (MK-1439), 294 a novel nonnucleoside reverse transcriptase inhibitor with distinct mutation development 295 pathways. Antimicrob Agents Chemother 2015; 59: 590-8. 
296 14. Smith SJ, Pauly GT, Akram A, et al. Rilpivirine and Doravirine Have Complementary 297 Efficacies Against NNRTI-Resistant HIV-1 Mutants. J Acquir Immune Defic Syndr 2016; 72: 48529891.

299 15. Soulie C, Santoro M, Charpentier C, et al. Rare occurrence of doravirine resistance300 associated mutations in HIV-1-infected treatment-naive patients. J Antimicrob Chemother $301 \quad 2019 ; 74: 614-7$.

302 16. Johnson $M$, Kumar P, Molina J-M, et al. Switching to Doravirine/Lamivudine/Tenofovir 303 Disoproxil Fumarate (DOR/3TC/TDF) Maintains HIV-1 Virologic Suppression Through 48 304 Weeks: Results of the DRIVE-SHIFT Trial. J Acquir Immune Defic Syndr 2019; 81 (4): 463-472.

305 17. Sterrantino G, Borghi V, Callegaro AP, et al. Prevalence of predicted resistance to 306 doravirine in HIV-1-positive patients after exposure to non-nucleoside reverse transcriptase 307 inhibitors. Int J Antimicrob Agents 2019; 53: 515-9. 


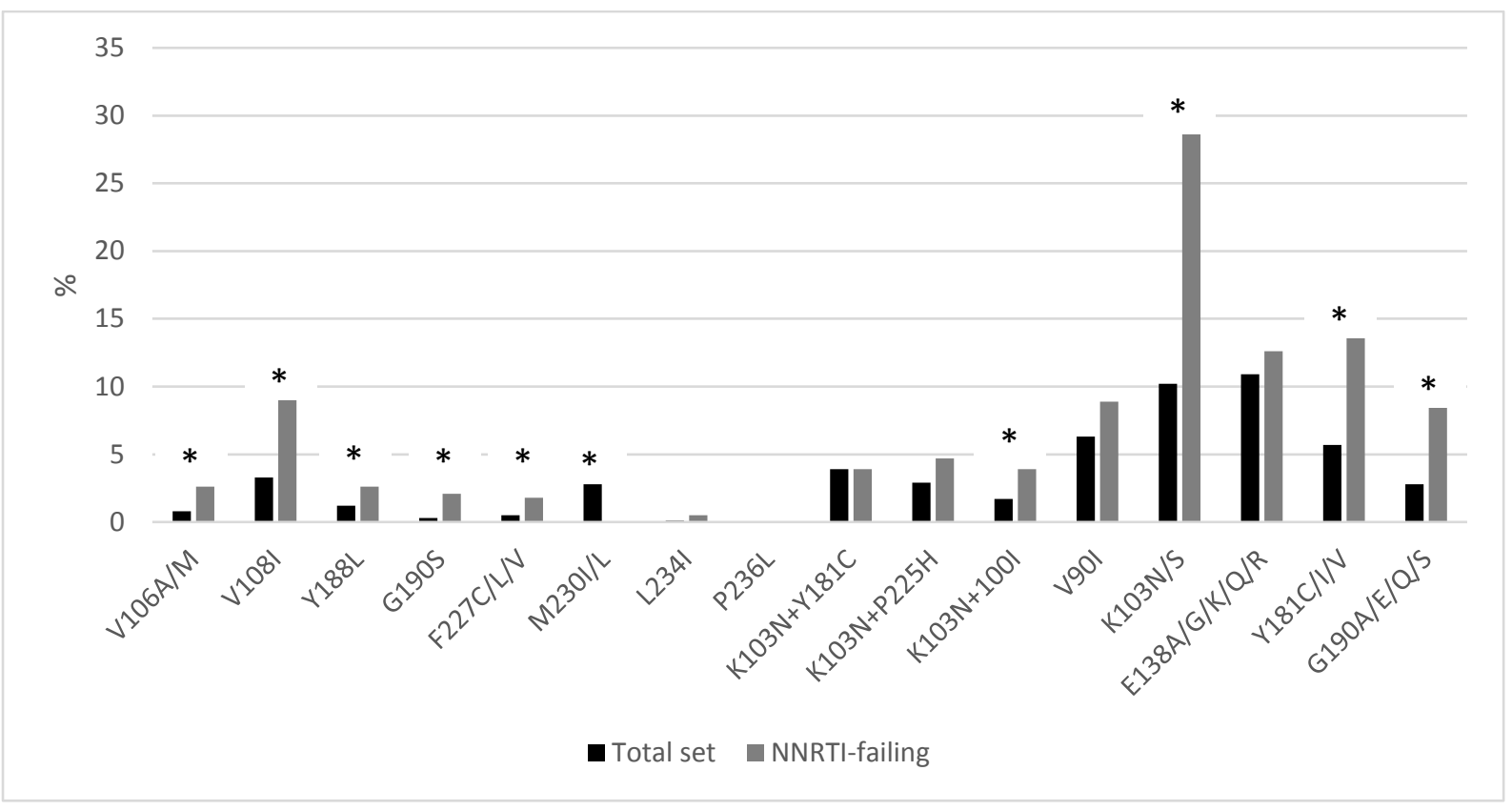

314 Figure 1: Prevalence of RT sequences with at least one individual doravirine or other NNRTI $315(>8 \%)$ resistance associated mutations in the total set (black) and in the NNRTI-failing group 316 (grey)

$317 * P<0.05:$ statistically different between total set and NNRTI-failing group 

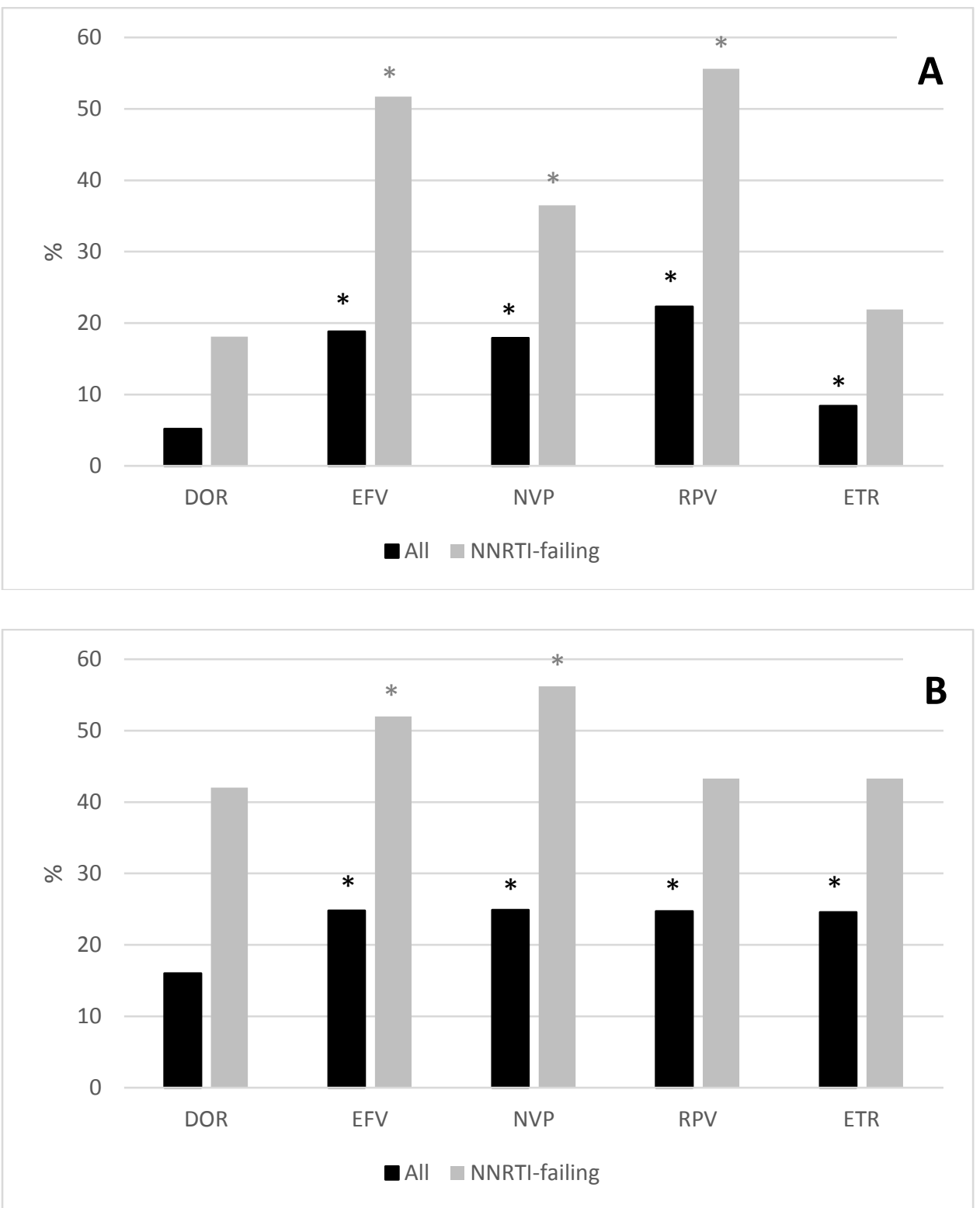

Figure 2: Percentage of RT sequences associated with NNRTI resistance in the whole data set and in the group of NNRTI-failing patients according to the ANRS (A) or Stanford (B) algorithm

328 DOR: doravirine, EFV: efavirenz, NVP: nevirapine, RPV: rilpivirine, ETR: etravirine ${ }^{*} \mathrm{P}<0.001$ : statistically different from Doravirine 